\title{
Experimental Induction of Isolated Lower Esophageal Sphincter Relaxation in Anesthetized Opossums
}

\author{
William G. Paterson, Satish Rattan, and Raj K. Goyal \\ Harvard Digestive Diseases Center, Charles A. Dana Research Institute, and Thorndike Laboratory, Division \\ of Gastroenterology, Beth Israel Hospital and Harvard Medical School, Boston, Massachusetts 02215
}

\begin{abstract}
Isolated lower esophageal sphincter (LES) relaxation, defined as a transient sphincteric relaxation unaccompanied by esophageal peristalsis, has been shown to precede most episodes of gastroesophageal reflux in humans. We studied the genesis of isolated LES relaxation in anesthetized opossums by observing the response of four components of the deglutition reflex (mylohyoid electrical activity, pharyngeal contraction, esophageal peristalsis, and LES relaxation) to pharyngeal tactile stimulation, electrical stimulation of superior laryngeal nerve (SLN) afferents or cervical vagal efferents, and to balloon distention of the esophageal body. A single pharyngeal stroking evoked isolated LES relaxation in $56 \%$ of 160 instances. The proportion of isolated relaxations in response to SLN electrical stimulation varied inversely with the stimulus frequency, occurring in $64 \%$ of the responses at $5 \mathrm{~Hz}$ and $4 \%$ of the responses at $30 \mathrm{~Hz}$. A full fourcomponent deglutition sequence was most likely to occur at the higher frequencies of SLN electrical stimulation. Esophageal balloon distention elicited isolated LES relaxations or no response at low distending volumes, whereas at higher volumes LES relaxation and esophageal contraction predominated. Isolated LES relaxation had significantly less magnitude than relaxations accompanied by esophageal contractions.

Bilateral cervical vagotomy abolished all LES and esophageal body responses induced by pharyngeal stroking and SLN stimulation, and rendered the esophageal body and LES less responsive to small volumes of distention. Vagal efferent stimulation produced isolated LES relaxation at lower frequency stimulation and LES relaxation with esophageal contractions at higher frequency stimulation. These studies show that isolated LES relaxation represents incomplete expression of either the deglutitive reflex or the peripheral reflex mediating secondary peristalsis.
\end{abstract}

\section{Introduction}

Lower esophageal sphincter (LES) ${ }^{1}$ relaxation accompanies both primary and secondary peristaltic contractions in the esophageal

A preliminary report of this work was presented at the Annual Meeting of the American Gastroenterological Association in New York, NY, 13 May 1985, and has appeared in abstract form (1984. Gastroenterology. 88:1533).

Address reprint requests to Dr. Goyal, Gastroenterology Division, Beth Israel Hospital, 330 Brookline Ave., Boston, MA 02215.

Received for publication 8 September 1985.

1. Abbreviations used in this paper: EMG, electromyogram; LES, lower esophageal sphincter; SLN, superior laryngeal nerve.

J. Clin. Invest.

(c) The American Society for Clinical Investigation, Inc. 0021-9738/86/04/1187/07 \$1.00

Volume 77, April 1986, 1187-1193 body, thereby allowing esophageal contents to pass into the stomach. The LES relaxation associated with primary peristalsis is mediated by inhibitory vagal efferent nerves (1), whereas the LES relaxation accompanying secondary peristalsis can be effected by intramural neuromuscular mechanisms $(2,3)$.

Transient relaxation of the LES, however, has been shown to occur without accompanying peristaltic contraction in the esophagus (4-7). Such isolated LES relaxation, also termed inappropriate LES relaxation (4), is believed to be of considerable importance, as it may play an important role in the pathogenesis of reflux esophagitis $(4,5)$. However, it is not known if such isolated LES relaxations are nonphysiological phenomena or if they represent part of a spectrum of physiological responses to swallowing and other reflex activities.

The present investigation was carried out to examine the possible mechanisms of induction of isolated or inappropriate LES relaxation. These studies show that LES relaxation can be activated in isolation by stimuli which are below threshold for eliciting the full deglutition or secondary peristalsis reflex.

\section{Methods}

Experiments were performed in 10 adult opossums (Didelphis virginiana) of either sex and weighing between 2.4 and $4.1 \mathrm{~kg}$. Animals were fasted for $12-16 \mathrm{~h}$ before the experiment. Anesthesia was induced with intraperitoneal pentobarbital sodium $(40 \mathrm{mg} / \mathrm{kg})$ and maintained with a constant intravenous infusion ( $15-20 \mathrm{mg} / \mathrm{kg}$ per $\mathrm{h}$ ) using a syringe pump (model 341A; Sage Instruments Div., Orion Instruments, Inc., Cambridge, MA). The animals were strapped supine to an animal board and kept warm with a heating pad. An endotracheal tube was placed and the animals were ventilated using a small animal respirator (model 661; Harvard Apparatus Co., Inc., The Ealing Corp., S. Natick, MA) at a rate and tidal volume determined by the weight of the animal according to the manufacturer's instructions. A brachial or femoral artery was cannulated for constant blood pressure recording.

The mylohyoid muscle was exposed in the neck and a bipolar electrode implanted in it. Electromyogram (EMG) activity of this muscle, which corresponds to the onset of deglutition (8), was recorded on an R711 dynograph (Beckman Instruments Inc., Fullerton, CA).

The motor activity of the esophagus was monitored with an assembly of catheters which were continuously perfused with bubble-free water at $0.33 \mathrm{ml} / \mathrm{min}$. The catheter assembly consisted of six polyvinyl tubes $(0.8$ $\mathrm{mm}$ i.d., $1.16 \mathrm{~mm}$ o.d.) which were glued together with tetrahydrofuran. A larger diameter tube ( $3 \mathrm{~mm}$ o.d.), to be used for pinning the assembly, was glued between the other tubes in the distal $5 \mathrm{~cm}$. One tube was left open at its distal end and was used to record gastric pressure. The others had 1-mm diameter side openings and the part distal to each opening was sealed. Two of the catheters had openings located $2 \mathrm{~cm}$ proximal to the tip of the assembly. The other catheters had openings 3, 7, and 20.5 $\mathrm{cm}$ from the tip. The assembly was introduced into the opossum esophagus by mouth and anchored as described previously (9) so that the openings located $2 \mathrm{~cm}$ from the tip recorded the LES pressure. The catheters were connected to pressure transducers (Statham P23 Db; Gould Statham Instruments Inc., Hato Rey, Puerto Rico), the outputs of which were displayed on an R711 dynograph (Beckman Instruments, Inc.). Pressures were recorded continuously from the stomach, LES, and phar- 
ynx as well as from the smooth muscle portion of the esophageal body 1 and $5 \mathrm{~cm}$ above the LES. Confirmation that the opening $20.5 \mathrm{~cm}$ from the assembly tip was monitoring pharyngeal activity was obtained by ensuring that this opening was above the upper esophageal high pressure zone in all animals. A gastrostomy tube was placed in order to continuously drain the stomach of accumulated fluid.

The deglutition reflex was elicited by pharyngeal stroking and electrical stimulation of the superior laryngeal nerve (SLN). Secondary peristalsis was evoked by balloon distention of the esophageal body. Pharyngeal stroking was done using a moistened cotton tip applicator. In order to standardize the stimulus and avoid movement artifact in the mylohyoid EMG, a small plastic tube was fixed in the mouth so that the applicator could be passed through this and applied directly to the posterior pharyngeal mucosa without contacting other tissues en route. The cotton tipped applicator was stroked only once, but firmly, over a small area of the posterior pharynx. Twenty observations were made in each of eight animals, with the interstimulus interval kept at at least $1 \mathrm{~min}$. In four animals sham pharyngeal stroking was performed as well. This consisted of lightly touching the applicator to the animals' tongues.

Electrical stimulation of the SLN was performed as follows. A midline incision was made in the neck and the right SLN was located and transected. The SLN contains mainly afferent fibers, and when stimulated electrically will evoke the deglutition reflex (10). The central end of this nerve was put on a bipolar $\mathrm{Ag}-\mathrm{AgCl}_{2}$ electrode for stimulation. Warm mineral oil and mineral oil-impregnated gauze were placed around the nerve and electrode to maintain nerve viability and minimize current leak. Stimuli were delivered by a stimulator (model S1 1A; Grass Instrument Co., Quincy, MA) through a constant current stimulus isolation unit (Grass Instrument Co., model PSIU6). The stimulus consisted of square wave pulses of $3-\mathrm{mA}$ current intensity, $0.5-\mathrm{msec}$ pulse duration, and 1-s train duration. The stimulus was presented at frequencies of 5 , $10,15,20,25$, and $30 \mathrm{~Hz} .15$ observations were made at each stimulus frequency in each of five animals. Stimuli were applied at intervals of at least $1 \mathrm{~min}$.

For the balloon distention experiments, the manometry tube was modified by attaching a polyvinyl tube with a small balloon at its tip so that the balloon was positioned $7 \mathrm{~cm}$ above the LES. Using a calibrated syringe, the balloon was inflated quickly with air, left for $2 \mathrm{~s}$, and then deflated. Distending volumes of $0.25,0.50,0.75,1.00,1.25,1.50,1.75$, and $2.00 \mathrm{~cm}^{3}$ were used in a random fashion. Eight observations at each balloon volume were made in each of five animals.

Electrical stimulation of the vagal efferent fibers was performed in three animals. The right vagus nerve was transected in the neck, and the distal end was placed on the stimulating electrode and immersed in warm mineral oil. The left vagus nerve remained intact. The nerve was stimulated with square wave pulses of 5-mA current intensity, 0.5-msec pulse duration, 1-s train duration, and frequencies of $1,2,5,10$, and $20 \mathrm{~Hz}$. Five observations were made at each frequency in each animal.

The influence of bilateral cervical vagotomy on LES responses to pharyngeal stimulation, electrical stimulation of the SLN, and esophageal distention was investigated. The vagi were isolated in the neck and before sectioning were identified physiologically by noting the characteristic cardiovascular and esophageal responses to electrical stimulation. The ends of the sectioned vagi were placed in warm mineral oil to prevent drying. Observations were begun $15 \mathrm{~min}$ after vagotomy.

A complete deglutition motor response consisted of EMG activity in the mylohyoid muscle, pharyngeal contraction, esophageal peristaltic contraction, and lower esophageal sphincter relaxation. The occurrence of these four components in isolation or in combination with other components was determined.

Responses were analyzed quantitatively and qualitatively by a blinded observer. LES pressure was referenced to simultaneously measured intragastric pressure. The magnitude of the LES relaxation was quantified by considering a fall in LES pressure to intragastric pressure as $100 \%$ relaxation. The duration of LES relaxation was measured from the onset of the abrupt descent in LES pressure to the point where LES pressure returned to prerelaxation baseline.
Data were analyzed using paired and unpaired Student's $t$ tests (11) and expressed as mean of the means in each animal $\pm \mathrm{SE}$.

\section{Results}

Patterns of responses. The various patterns of responses evoked by pharyngeal stimulation, SLN afferent or vagal efferent stimulation, and balloon distention are summarized in Fig. 1. These consisted of $(a)$ a full four-component response involving mylohyoid activity, pharyngeal and esophageal contraction, and LES relaxation; $(b)$ esophageal peristalsis and LES relaxation without mylohyoid activity or pharyngeal contraction; (c) mylohyoid activity and pharyngeal contraction with LES relaxation but without esophageal peristaltic contractions; $(d)$ mylohyoid activity with LES relaxation but without pharyngeal or esophageal contractions, and $(e)$ LES relaxation alone without mylohyoid, pharyngeal, or esophageal contractions. Note that LES relaxation was the most consistent component of all the response patterns. Rarely, mylohyoid activity without any other response was observed with pharyngeal stimulation. Moreover, while pharyngeal and SLN stimulation could evoke any of the above patterns of responses, balloon distention and vagal efferent stimulation did not evoke responses involving mylohyoid activity or pharyngeal contractions.

Responses to pharyngeal tactile stimulation. The incidence of the different patterns of responses to pharyngeal stroking are shown in Table I. In these anesthetized animals, repetitive or vigorous pharyngeal stroking was usually required to predictably

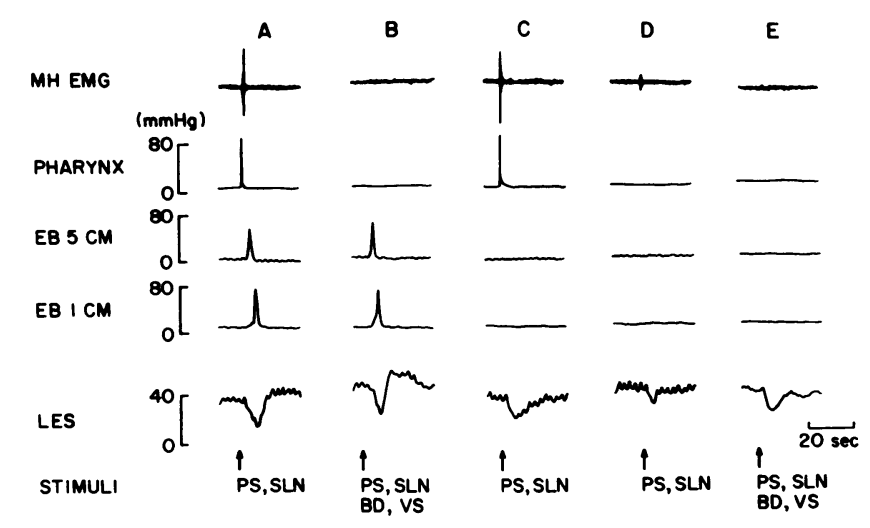

Figure 1. Different responses observed after pharyngeal stroking (PS), SLN stimulation, balloon distention $(B D)$ of the esophageal body, and vagal efferent stimulation $(V S)$. LES relaxation accompanies all the response types (except for a few instances of isolated mylohyoid $(M H)$ EMG activity). Pharyngeal stroking and SLN stimulation evoked the entire spectrum of responses, whereas balloon distention and vagal stimulation elicited either isolated LES relaxation or LES relaxation associated with esophageal body contractions. $E B 1 \mathrm{~cm}, E B 5 \mathrm{~cm}$, esophageal body 1 and $5 \mathrm{~cm}$ above the LES. Pressure scale on left references atmospheric pressure to 0 . $(A)$ Shows full four-component deglutition response consisting of mylohyoid activity, pharyngeal contraction, esophageal body peristalsis, and LES relaxation. (B) Shows esophageal body contraction and LES relaxation without mylohyoid activity or pharyngeal contraction. $(C)$ Shows mylohyoid activity and pharyngeal contraction associated with LES relaxation but failed esophageal body contraction. $(D)$ Shows LES relaxation associated with low amplitude mylohyoid EMG activity but no pharyngeal or esophageal body contraction. $(E)$ Shows LES relaxation only. 
Table I. Incidence of Different Motor Deglutitive Responses to a Single Pharyngeal Stroking

\begin{tabular}{lc}
\hline Type of response & No. (\%) \\
\hline Full four-component response & $16(10)$ \\
LES relaxation with esophageal contraction only & $15(9.4)$ \\
LES relaxation with mylohyoid activity and & \\
$\quad$ pharyngeal contraction only & $2(1.2)$ \\
LES relaxation and mylohyoid activity only & $31(19.4)$ \\
LES relaxation only & $57(35.6)$ \\
Mylohyoid activity only & $8(5)$ \\
No response & $31(19.4)$ \\
Total & $160(100)$
\end{tabular}

elicit all four components of the deglutition reflex. With the single pharyngeal stroking method used, complete four-component swallowing sequences were seen in only $10 \%$ of $160 \mathrm{ob}-$ servations. The most common response, seen in $35.6 \%$, was LES relaxation without any other recognizable evidence of deglutition activity. LES relaxation associated with mylohyoid EMG activity but without other components of deglutition occurred in another $19.4 \%$, and LES relaxation associated with pharyngeal and mylohyoid responses but no esophageal contractions occurred in $1.2 \%$. Thus, responses which consisted of LES relaxation without esophageal contractions occurred in over one-half $(56.2 \%)$ of the observations. LES relaxation associated with contraction waves in the distal esophageal body but no pharyngeal contraction occurred 15 times $(9.4 \%)$. In no instance did pharyngeal or esophageal body contractions occur without associated LES relaxation. Of the 80 sham stimuli applied, only 1 was interpreted as having possibly evoked a response.
The magnitude of LES relaxation was significantly less when LES relaxation occurred alone or in association with mylohyoid EMG or pharyngeal contraction than when relaxation was part of a complete four-component swallowing sequence (34.5\% vs. $86.0 \% ; P<0.001$ ), or associated with esophageal but no pharyngeal contraction $(34.5 \%$ vs. $76.9 \% ; P<0.001)$. There was an overlap in the range of magnitude of isolated LES relaxations $(10-80 \%)$ and relaxations associated with the four-component response $(58-100 \%)$. $7 \%$ of the isolated LES relaxations were $>58 \%$. The mean duration of LES relaxation was also less for isolated LES relaxations than for those associated with the fourcomponent response $(5.3 \pm 0.4$ vs. $8.4 \pm 0.8 \mathrm{~s} ; P<0.01)$. The range of durations of isolated LES relaxations (2.5-10 s) overlapped the range seen with the four-component response (5.5-11 s). $13 \%$ of the isolated LES relaxations had durations within the range of the four-component response. The difference between the magnitude of LES relaxation associated with the complete four-component response and that associated with esophageal but no pharyngeal contraction was not statistically significant (86.0\% vs. $76.9 \% ; P>0.05)$.

Pharyngeal stroking after bilateral cervical vagotomy ( $50 \mathrm{ob}-$ servations in three animals) did not evoke esophageal peristalsis or LES relaxation, suggesting that the esophageal peristalsis and LES relaxation evoked by pharyngeal stimulation are mediated centrally via the vagus nerve. As expected, mylohyoid and pharyngeal responses were preserved because efferent fibers to these muscles originate from the trigeminal nerve and from the vagus at a point proximal to the site of vagotomy, respectively (12).

Responses to superior laryngeal nerve afferent stimulation. The spectrum of response to SLN afferent stimulation was similar to that elicited by pharyngeal stroking (Fig. 1). The various types of response observed were dependent on the SLN stimulus frequency used to evoke the response. Table II shows the incidence of different responses at varying SLN stimulus frequencies. Note that the incidence of a positive response increased as the SLN

Table II. Patterns of Responses to Different Frequencies of SLN Afferent Stimulation

\begin{tabular}{|c|c|c|c|c|c|c|}
\hline \multirow[b]{2}{*}{ Type of Response } & \multicolumn{6}{|c|}{ SLN stimulus frequency } \\
\hline & $5 \mathrm{~Hz}$ & $10 \mathrm{~Hz}$ & $15 \mathrm{~Hz}$ & $20 \mathrm{~Hz}$ & $25 \mathrm{~Hz}$ & $30 \mathrm{~Hz}$ \\
\hline & No. $\%^{*}$ & No. $\%$ & No. $\%$ & No. $\%$ & No. $\%$ & No. $\%$ \\
\hline Full four-component response & $3(27.3)$ & $5(15.2)$ & $13(25.5)$ & $41(62.1)$ & $57(78.1)$ & $62(83.8)$ \\
\hline $\begin{array}{l}\text { LES relaxation with esophageal } \\
\text { contraction only }\end{array}$ & $1(9.1)$ & $0(0)$ & $2(3.9)$ & $3(4.5)$ & $1(1.4)$ & $2(2.7)$ \\
\hline $\begin{array}{l}\text { LES relaxation with mylohyoid } \\
\text { activity and pharyngeal }\end{array}$ & & & & & & \\
\hline contraction only & $0(0)$ & $6(18.2)$ & $11(21.6)$ & $10(15.2)$ & $7(9.6)$ & $7(9.5)$ \\
\hline LES relaxation and mylohyoid & & & & & & \\
\hline activity only & $1(9.1)$ & $8(24.2)$ & $6(11.8)$ & $7(10.6)$ & $3(4.1)$ & $3(4.1)$ \\
\hline LES relaxation only & $6(54.5)$ & $14(42.4)$ & $19(37.3)$ & $5(7.6)$ & $5(6.8)$ & $0(0)$ \\
\hline Total number of positive & & & & & & \\
\hline responses & 11 & 33 & 51 & 66 & 73 & 74 \\
\hline No response & 64 & 41 & 24 & 9 & 2 & 1 \\
\hline Total observations & 75 & $75 \dagger$ & 75 & 75 & 75 & 75 \\
\hline
\end{tabular}

* Refers to percent of positive responses. $¥$ Not included in table is one observation at SLN stimulus frequency of $10 \mathrm{~Hz}$ that resulted in isolated mylohyoid EMG activity. 
stimulus frequency was increased. At $5 \mathrm{~Hz}$ a response was evoked in only 11 of 75 observations, whereas at $30 \mathrm{~Hz}$ a response occurred in 74 of 75 observations.

The incidence of the complete four-component response increased with increasing frequency of SLN stimulation, whereas the incidence of isolated LES relaxation decreased as the stimulus frequency was increased. $27 \%$ of the responses to $5 \mathrm{~Hz}$ were complete swallowing sequences as opposed to $84 \%$ of the responses to $30 \mathrm{~Hz}$. Conversely, LES relaxation alone or associated with mylohyoid EMG activity occurred in $63.6 \%$ of the 11 responses to $5 \mathrm{~Hz}$, whereas it occurred in only $4.1 \%$ of the 74 responses to $30 \mathrm{~Hz}$.

Fig. 2 illustrates the effect of varying SLN afferent stimulus frequency on the isolated LES relaxation and full four-component response types with respect to three variables, namely, the number of animals responding, the mean number of responses per responding animal, and the mean percent LES relaxation per response. The mean magnitude of isolated LES relaxation is significantly less than the relaxation seen with the four-component response at all SLN stimulus frequencies. Isolated LES relaxations ranged in magnitude from $10-79 \%$, whereas relaxations associated with the four-component response ranged from $27-100 \% .26 \%$ of the isolated LES relaxations fell into the range seen with the four-component response. The mean duration of

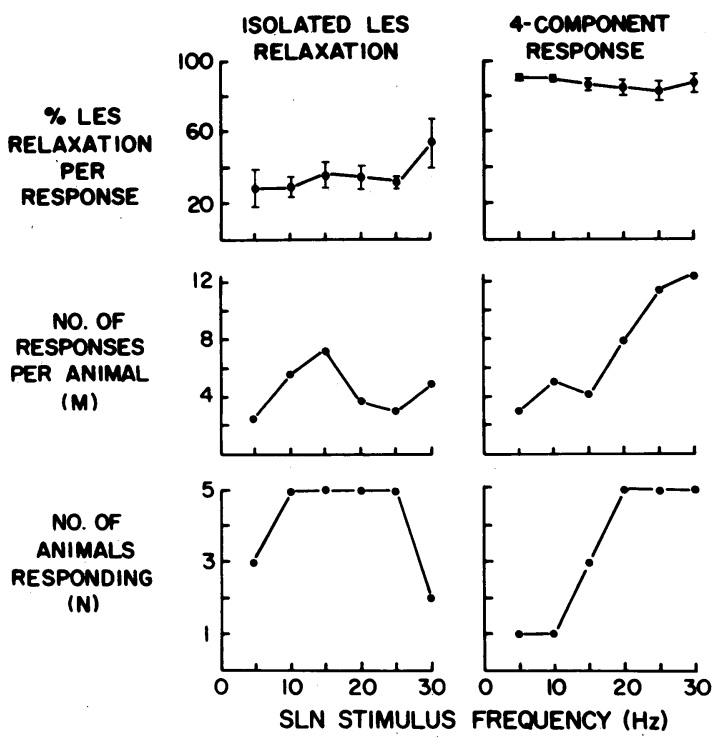

Figure 2. Comparison of two response types, namely, isolated LES relaxation and full four-component swallowing sequence with respect to three variables: $(a)$ number of animals responding $(N)$ out of a total of five; $(b)$ mean number of responses per responding animal $(M)$; and (c) mean percent LES relaxation per response (mean of means in each animal $\pm \mathrm{SE}$ ). For the isolated LES relaxation response, $N$ and $M$ tend to be lowest at the high and low extremes of SLN stimulus frequency and highest at the mid-range of frequencies, whereas for the full fourcomponent response $N$ and $M$ increase with increasing stimulus frequency. The mean percent LES relaxation is significantly lower for the isolated LES relaxation response than for the full four-component response at all SLN stimulus frequencies tested $(P<0.05$ at $30 \mathrm{~Hz}$ and $P<0.001$ at $5,10,15,20$, and $25 \mathrm{~Hz}$ ). There is no statistically significant difference between the magnitude of LES relaxation at the different SLN stimulus frequencies for the full four-component type response. For the isolated LES relaxation response, the magnitude of LES relaxation at 5 and $10 \mathrm{~Hz}$ was significantly less than that at $30 \mathrm{~Hz}$ $(P<0.05)$. the isolated LES relaxations evoked by SLN stimulation was not statistically different from that of relaxations associated with the full four-component response $(5.9 \pm 0.7 \mathrm{~s}$ vs. $7.3 \pm 0.7 \mathrm{~s})$. Isolated LES relaxations ranged in duration from 3 to $10 \mathrm{~s}$, whereas relaxations associated with the four-component response ranged from 3 to $13 \mathrm{~s} .79 \%$ of the isolated LES relaxations had durations in the range of the four-component response.

In two animals, 10 observations were made at each SLN stimulus frequency after bilateral cervical vagotomy. No LES relaxation or esophageal body contractions were evoked after the vagus nerves were cut. Mylohyoid and pharyngeal responses were maintained.

Responses to balloon distention of esophageal body. Responses to balloon distention consisted of either LES relaxation alone or LES relaxation associated with contraction recorded in one or both of the distal esophageal leads (Fig. 1). In no instance did balloon distention evoke pharyngeal contraction or mylohyoid activation. Esophageal body contractions were not seen without associated LES relaxation. The presence or absence of a response was dependent on the magnitude of balloon distention of the esophagus, in that larger distending volumes were more likely to evoke a response. At $0.25,0.50$, and $0.75 \mathrm{~cm}^{3}$ of balloon distention, responses were seen in 17, 35, and 39 of 40 observations, respectively. Responses were always evoked by balloon distending volumes of $1.0 \mathrm{~cm}^{3}$ or greater. The type of response elicited was also dependent on the volume of distention used. As shown in Fig. 3, when a response did occur with low volumes of balloon distention, it was most likely to be an isolated LES relaxation $\left(82 \%\right.$ incidence with $\left.0.25 \mathrm{~cm}^{3}\right)$, whereas at high volumes the incidence of isolated LES relaxation declined and secondary peristaltic responses predominated.

The magnitude of LES relaxation with balloon distention depended on the type of response evoked. It was significantly less with isolated LES relaxation than with LES relaxation associated with esophageal body contraction $(28.0 \pm 7.7 \%$ vs. $52.2 \pm 4.9 \% ; P<0.001)$. The magnitude of the isolated LES relaxations ranged from 7 to $65 \%$, whereas relaxations associated with the full secondary peristalsis response ranged from 25 to $97 \%$. One-quarter of the isolated LES relaxations evoked by bal-

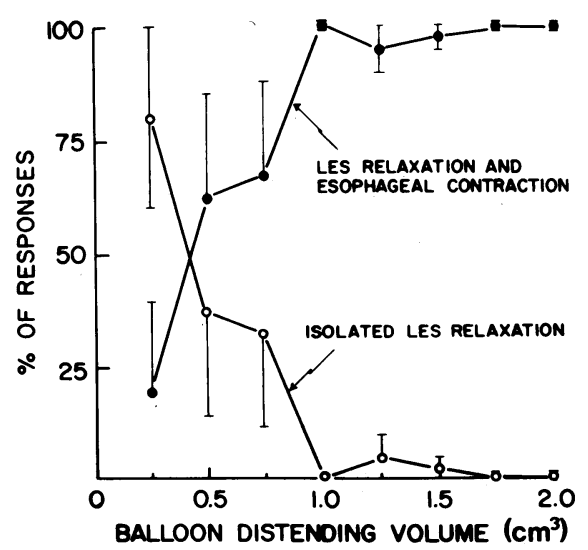

Figure 3. Relationship between esophageal balloon distending volume and incidence of different responses. Data expressed as mean of means in each animal \pm SE. Note that isolated LES relaxation predominates at low distending volumes and the incidence steadily declines as distending volume is increased, whereas the opposite occurs with LES relaxation associated with esophageal body contraction. 
loon distention fell into the magnitude range of the LES relaxations associated with secondary peristalsis. The balloon volume used to evoke a response did not influence the magnitude of the LES relaxation in that sphincteric relaxations associated with isolated LES relaxation were of similar magnitude at all distending volumes used. The same held true for the LES relaxations accompanied by esophageal body contraction. The mean duration of the isolated LES relaxations was significantly less than that seen with LES relaxations accompanying secondary peristalsis $(5.1 \pm 0.5 \mathrm{~s}$ vs. $7.5 \pm 0.2 \mathrm{~s} ; P<0.01)$. Durations for the isolated LES relaxations ranged from 2 to $8.5 \mathrm{~s}$ and for relaxations associated with secondary peristalsis from 3.5 to $11.5 \mathrm{~s}$. $55 \%$ of the isolated LES relaxations were in the duration range of the LES relaxations accompanying secondary peristalsis.

Bilateral cervical vagotomy did not abolish esophageal responses to balloon distention. However, as seen in Fig. 4, at the low volumes of balloon distention the volume-response curve is shifted to the right, indicating that the esophagus is less sensitive to small degrees of distention after vagotomy. The relationship of response type to volume of balloon distention was not changed after vagotomy and the magnitude of LES relaxation was no different from that seen with a similar response pre-vagotomy.

Responses to vagal efferent stimulation. LES relaxation with or without esophageal body contractions was evoked by all frequencies of cervical vagal efferent stimulation used. Vagal stimulation did not elicit pharyngeal contraction or mylohyoid EMG activity. In only one trial (at $1 \mathrm{~Hz}$ ) was there failure to evoke a response. The type of response was dependent on the frequency of stimulation used as depicted in Fig. 5. At 1 and $2 \mathrm{~Hz}$ virtually all the responses were isolated LES relaxations, whereas at the higher frequencies the incidence of isolated LES relaxation progressively declined as LES relaxation accompanied by esophageal body contraction took over.

The magnitude of LES relaxation was also dependent on the type of response seen. The average relaxation occurring with isolated LES relaxation for all frequencies combined was $35.2 \pm 5.0 \%$ (range, 6-63\%), whereas with LES relaxation accompanied by esophageal body contraction the mean relaxation was $76.8 \pm 4.2 \%$ (range, $25-97 \% ; P<0.001$ ). $25 \%$ of the isolated LES relaxations evoked by vagal stimulation fell into the mag-

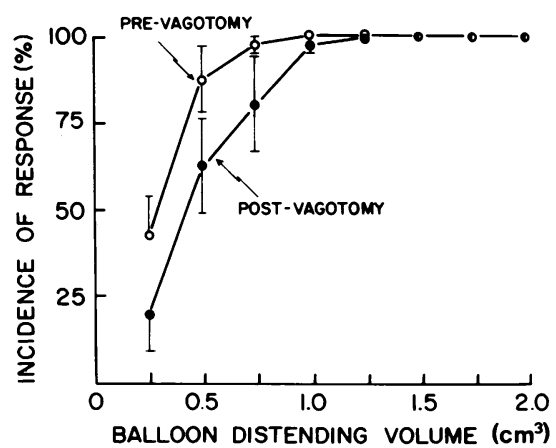

Figure 4. Influence of bilateral cervical vagotomy on esophageal response to balloon distention. The incidence of response is expressed as mean of means in each animal $\pm \mathrm{SE}$. At the lower volumes of balloon distention, the volume response curve is shifted to the right postvagotomy, indicating a decreased sensitivity of the esophagus to distention. Differences in the incidence of response using the paired $t$ test are significant for $0.25 \mathrm{~cm}^{3}(P<0.001)$ and $0.50 \mathrm{~cm}^{3}(P<0.05)$ but not for $0.75 \mathrm{~cm}^{3}$ or higher volumes.

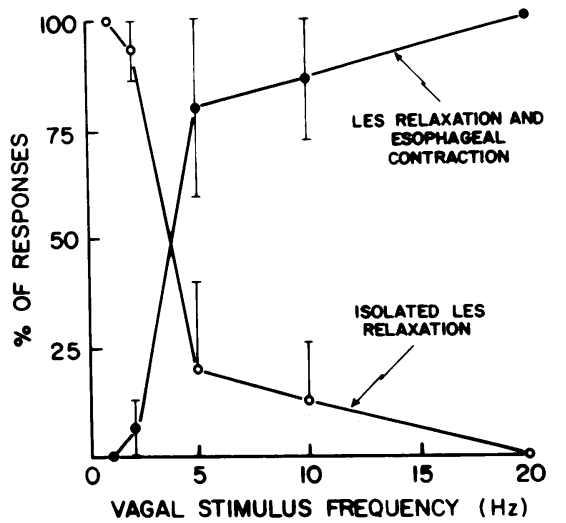

Figure 5. Influence of vagal efferent stimulus frequency on incidence of different responses. Data expressed as mean of means in each animal \pm SE. Note that isolated LES relaxation predominates at low frequency vagal efferent stimulation, whereas LES relaxation and esophageal body contraction predominate at high frequency stimulation.

nitude range of the LES relaxations associated with esophageal body contractions. The mean duration of the isolated LES relaxations was significantly less than those associated with esophageal contractions $(3.7 \pm 0.1 \mathrm{~s}$ vs. $6.8 \pm 0.4 \mathrm{~s} ; P<0.01)$. Durations ranged from 2.5 to $5.5 \mathrm{~s}$ for isolated LES relaxations, and from 4.5 to $9.0 \mathrm{~s}$ for LES relaxations associated with esophageal contractions. $17 \%$ of the isolated LES relaxations evoked by vagal efferent stimulation fell into the duration range of the full response.

Increasing frequency of vagal efferent stimulation tended to increase the magnitude of LES relaxation. This was true for both types of response.

\section{Discussion}

These studies show that transient isolated LES relaxation, that is, sphincteric relaxation unaccompanied by esophageal peristaltic contraction, may represent a fragmented efferent component of the centrally mediated deglutition reflex responsible for primary peristalsis, or the peripheral reflex involved in secondary peristalsis.

The motor component of the deglutition reflex is generally divided into oropharyngeal, esophageal, and gastroesophageal phases (10). Although all of these three phases are normally evoked by swallowing, it is known that some of these components can occur in isolation. For example, the oropharyngeal phase in the absence of esophageal peristalsis normally occurs during rapid successive swallows, as in drinking $(10,13)$, and can also occur in response to single swallows (14-16). On the other hand, esophageal peristalsis in the absence of oropharyngeal contractions normally occurs during secondary peristalsis induced by esophageal distention (10).

The dissociation of LES relaxation from pharyngeal or esophageal peristaltic contractions has not been previously investigated in detail. Doty speculated that LES relaxation and esophageal peristalsis may fail to occur in response to swallows, although he had no supportive data (10). In our study pharyngeal and/or esophageal contraction never occurred in the absence of LES relaxation. The converse, that is relaxation of the LES without pharyngeal or esophageal contraction, has been described in humans $(4,5,7)$ and occurred frequently in our animals. 
It has been suggested but not documented that these isolated LES relaxations in humans may be related to the deglutition reflex $(4,6,17)$. We observed that elicitation of full deglutition required vigorous pharyngeal stroking, whereas light single pharyngeal stroking usually evoked only LES relaxation. This suggested that isolated LES relaxation may be due to activation of the swallowing center by subthreshold sensory input. In support of this was the fact that although LES relaxation frequently occurred in isolation, pharyngeal and esophageal peristaltic contractions were always associated with LES relaxation. In order to quantitatively examine the influence of sensory stimulus intensity on the motor components of the deglutition reflex, the influence of SLN stimulation at different stimulus frequencies was examined. Low stimulus frequencies $(5$ and $10 \mathrm{~Hz}$ ) usually evoked either no response or isolated LES relaxation. At high stimulus frequencies the full four-component motor response was observed. This supported the view that the evoked isolated LES relaxations in our animal model are due to subthreshold activation of the deglutition reflex.

The mylohyoid muscle is generally considered to be the lead muscle of deglutition because it is the first muscle to show activity on swallowing (8). Therefore, mylohyoid muscle activity is often used as a marker for swallowing. In our studies, mylohyoid activity frequently occurred without pharyngeal or esophageal contraction in response to pharyngeal or SLN stimulation, suggesting that mylohyoid activity is a more sensitive marker of deglutitive activity than pharyngeal or esophageal contraction. Mylohyoid activity, however, was often associated with LES relaxation but no other motor components of deglutition, further suggesting that the evoked isolated LES relaxation represents a fragmented component of the deglutition reflex.

In many instances, tactile pharyngeal stimulation or SLN stimulation caused only LES relaxation without any other evidence of deglutitive activity. It appears, therefore, that LES relaxation is one of the most sensitive components of the deglutition reflex, and may represent the only recognizable manifestation of deglutition when the reflex is activated by low intensity stimuli.

When evoked by pharyngeal or SLN stimulation, isolated LES relaxation, like the esophageal component of the deglutition reflex, is mediated by vagal efferents, as bilateral vagotomy abolishes these responses. It has been suggested that there are separate inhibitory and excitatory vagal pathways which mediate deglutitive responses (13). The reason why sensory stimuli which are subthreshold for the full deglutition reflex produce isolated LES relaxation is not known, but may, however, be explained in one of two ways: (a) a subthreshold sensory stimulus may selectively activate the inhibitory pathway in the swallowing center without activating excitatory efferents, or $(b)$ inhibitory and excitatory pathways are equally activated but the peripheral neuromuscular mechanism may be more sensitive to low intensity inhibitory discharge than to low intensity excitatory discharge. Either of these two possibilities is compatible with our data showing selective activation of LES relaxation by low frequency vagal efferent stimulation. Recent electrophysiological studies, however, suggest that the short latency efferent fibers corresponding to the descending inhibition have a lower threshold for activation than the long latency fibers that correspond to esophageal contraction (18). This is consistent with the view that isolated LES relaxation may represent selective central activation of the inhibitory component of the deglutition reflex. Furthermore, it has been shown recently that low intensity electrical stimulation of the cat nucleus ambiguus or dorsal motor nucleus of the vagus readily elicits LES relaxation, whereas higher intensity stimulation is required to evoke esophageal body contractions (19).

Other reflex activities such as belching and vomiting are associated with LES relaxation without esophageal contractions. These reflexes may involve central neurons which are responsible for LES relaxation during swallowing. It is possible that afferent stimuli which are subthreshold for full vomiting or belching reflexes can produce isolated LES relaxation without any other motor manifestations. Further studies are needed to resolve these issues.

Esophageal distention produces esophageal peristaltic contractions and LES relaxation via intramural neuromuscular mechanisms $(2,3)$. Isolated LES relaxation, however, occurred with balloon distentions that were subthreshold for producing a full secondary peristaltic sequence. Our studies show that bilateral vagotomy increased the threshold of this reflex response, suggesting that vagus nerves exert a facilitatory influence on this reflex. This may be due to changes in esophageal diameter or wall compliance due to vagotomy. It appears more likely, however, that an afferent-efferent vagal loop somehow acts to increase the sensitivity of the secondary peristaltic reflex. It is of interest in this regard that gastric distention has recently been reported to facilitate the occurrence of isolated LES relaxation in humans (7).

Although some evoked isolated LES relaxations were of a magnitude comparable to that seen with the full primary or secondary peristaltic response, most of the isolated relaxations were of lesser duration and magnitude and were incomplete. Such incomplete LES relaxations do not abolish the gastroesophageal pressure barrier and are not associated with gastroesophageal reflux $(4,5)$. In contrast, isolated LES relaxations associated with gastroesophageal reflux in humans were complete, that is, the gastroesophageal pressure barrier was abolished $(4,5)$. Therefore, the relationship between incomplete isolated sphincter relaxation in the animal model and complete isolated sphincter relaxation in humans must remain speculative.

The completeness of isolated LES relaxation may be related to the intensity of afferent stimulation and the presence of facilitatory influences. We have observed that when multiple or more vigorous pharyngeal stroking causes isolated LES relaxation, the magnitude of the relaxation is much larger than when evoked by a single stroking. (Paterson, W. G., S. Rattan, and R. K. Goyal, unpublished observations). Furthermore, as is evident in Fig. 2, the magnitude of the isolated LES relaxation evoked by $30 \mathrm{~Hz}$ of SLN stimulation is significantly greater than that evoked by 5 or $10 \mathrm{~Hz}$. This suggests that more vigorous stimulation is responsible for the completeness of the isolated LES relaxation. It is possible that anesthesia in some way inhibited maximal isolated LES relaxation in our animal model, as in human studies isolated complete LES relaxations only occurred while the subject was awake or during arousal from sleep, but not during deep sleep (4). Perhaps most isolated LES relaxations in normal human subjects are also incomplete and only occasionally is a full relaxation produced which leads to gastroesophageal reflux. In patients with a hypotensive LES the incomplete sphincter relaxations may be enough to cause obliteration of the lower esophageal high pressure zone leading to gastroesophageal reflux. Alternatively, other facilitatory influences may be operative in producing a complete isolated LES relaxation (7).

In the present studies isolated LES relaxations were all 
evoked, whereas the stimulus for the isolated LES relaxations described in humans is not known. It is possible that these apparently spontaneous LES relaxations represent subthreshold activation of swallowing due to a collection of saliva in the oropharynx. Also, the manometry tube in the pharynx may be facilitating LES relaxations in the unanesthetized state.

In addition to its physiologic importance, isolated LES relaxation may be of considerable clinical interest in that episodes of isolated LES relaxation are associated with gastroesophageal reflux both in normal humans as well as in reflux esophagitis (5). It remains to be determined whether subthreshold activation of the peristalsis, vomiting, and belching reflexes, with or without other facilitatory influences, is responsible for the human isolated LES relaxations associated with gastroesophageal reflux.

\section{Acknowledgments}

The authors thank Marcia Thornhill for typing the manuscript, Dr. Jeffrey Crist and Dr. Ashok Sen Gupta for helpful suggestions, and Dr. Bernard Ransil for assistance in statistical analysis.

This work was supported in part by grant RR01032 from the General Clinical Research Centers Program of the Division of Research Resources, National Institutes of Health; and by grants AM31092 and Electrophysiology Core No. 49915 from National Institute of Arthritis, Metabolism, Digestive Diseases, and Kidney of the National Institutes of Health. Dr. Paterson is supported by a fellowship from the Medical Research Council of Canada and a Research Training Supplement from the American Gastroenterology Association.

\section{References}

1. Rattan, S., and R. K. Goyal. 1974. Neural control of the lower esophageal sphincter. Influence of the vagus nerves. J. Clin. Invest. 54: 899-906.

2. Ryan, J. P., W. J. Snape, and S. Cohen. 1977. Influence of vagal cooling on esophageal function. Am. J. Physiol. 232(2):E159-E164.

3. Tieffenbach, L., and C. Roman. 1972. Role de l'innervation extrinsèque vagale dans la motricité de l'oesophage à musculeuse lisse: étude electromyographique chez le chat et le babouin. J. Physiol. (Paris). 64:193-226.

4. Dent, J., W. J. Dodds, R. H. Friedman, T. Sekiguchi, W. J. Hogan, R. C. Arndorfer, and D. J. Petrie. 1980. Mechanism of gastroesophageal reflux in recumbent asymptomatic human subjects. J. Clin. Invest. 65: 256-267.

5. Dodds, W. J., J. Dent, W. J. Hogan, J. F. Helm, R. Hauser, G. K. Patel, and M. S. Egide. 1982. Mechanisms of gastroesophageal reflux in patients with reflux esophagitis. $N$. Engl. J. Med. 307:15471552.

6. Dodds, W. J., W. J. Hogan, J. F. Helm, and J. Dent. 1981. Pathogenesis of reflux esophagitis. Gastroenterology. 81:376-394.

7. Holloway, R. H., M. Hongo, K. Berger, and R. W. McCallum. 1985. Gastric distention: a mechanism for postprandial gastroesophageal reflux. Gastroenterology. 89:779-784.

8. Doty, R. W., and J. F. Bosma. 1956. An electromyographic analysis of reflex deglutition. J. Neurophysiol. (Bethesda). 19:44-60.

9. Goyal, R. K., and S. Rattan. 1976. Genesis of basal sphincter pressure: effect of tetrodotoxin on lower esophageal sphincter pressure in opossum in vivo. Gastroenterology. 71:62-67.

10. Doty, R. W. 1968. Neural organization of deglutition. In Handbook of Physiology. Alimentary Canal Motility. C. F. Code, editor. American Physiological Society, Washington, DC. 1861-1902.

11. Snedecor, G. W., and W. G. Cochran. 1976. Statistical Methods. Iowa State University Press, Ames, IA. 91-119.

12. Hwang, K., M. I. Grossman, and A. C. Ivy. 1948. Nervous control of the cervical portion of the esophagus. Am. J. Physiol. 154:343-357.

13. Goyal, R. K., and B. W. Cobb. 1981. Motility of the pharynx, esophagus, and esophageal sphincters. In Physiology of the Gastrointestinal Tract. L. R. Johnson, editor. Raven Press, New York. 359-391.

14. Siegal, C., and T. R. Hendrix. 1961. Evidence for the central mediation of secondary peristalsis in the esophagus. Bull. Johns Hopkins Hosp. 108:297-307.

15. Hollis, J. B., and D. O. Castell. 1975. Effect of dry swallows and wet swallows of different volumes on esophageal peristalsis. J. Appl. Physiol. 38:1161-1164.

16. Dodds, W. J., W. J. Hogan, D. P. Reid, E. T. Stewart, and R. C. Arndorfer. 1973. A comparison between primary esophageal peristalsis following wet and dry swallows. J. Appl. Physiol. 35:851-857.

17. Carlson, A. J., T. E. Boyd, and J. F. Pearcy. 1922. Studies on the visceral sensory nervous system XIV. The reflex control of the cardia and lower esophagus in mammals. Arch. Intern. Med. 30:409-433.

18. Gidda, J. S., and R. K. Goyal. 1984. Swallow-evoked action potentials in vagal preganglionic efferents. J. Neurophysiol. (Bethesda). 52:1169-1180.

19. Barone, F. C., D. M. Lombardi, H. S. Ormsbee III. 1984. Effects of hindbrain stimulation on lower esophageal sphincter pressure in the cat. Am. J. Physiol. 247:G70-G78. 